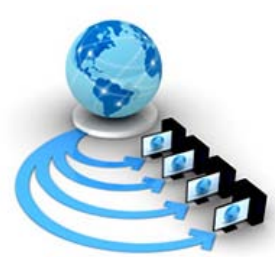

\title{
SECURE AND INTELLIGENT POWER MANAGEMENT SCHEMA FOR HETEROGENOUS DISTRIBUTED WIRELESS MULTIMEDIA SENSOR NETWORKS
}

\author{
Avinash Singh* \\ Department of CS \\ DDU Gorakhpur University \\ Gorakhpur, India
}

\author{
Surya Pratap Singh \\ Department of CS \\ DDU Gorakhpur University \\ Gorakhpur, India
}

\author{
Arvind Kumar Maurya \\ Department of CS \\ DDU Gorakhpur University \\ Gorakhpur, Indi
}

\author{
Upendra Nath Tripathi \\ Department of CS \\ DDU Gorakhpur University \\ Gorakhpur, India
}

\begin{abstract}
For wireless multimedia sensor networks, very large number of sensor nodes are being used having different types sensing capability in order to gather various types of data from different environments. For multimedia type applications, considering its huge data volume structure, it is necessary to provide reliable and fair protocols in order to meet its specific requirements of quality of service demands in regard to manage them. To persist the system lifetime of wireless multimedia sensor networks, it is necessary to perform adjustments to maintain transmission rate and to manage traffic congestion In addition, scheme needs an effectively control mechanism for handling different data transmission with maintained quality of service for system without compromising security services. In this paper, we propose a fuzzy system based efficient routing protocol for heterogeneous distributed sensor mobile ad-hoc networks technique that aims to improve routing protocols by considering both energy and security. We developed fuzzy logic controller that trace route by ant path search algorithm and implement security by using a novel hash function. Finally, we simulated and compared the proposed protocol with the some existing one where, analysis and verification results shows that proposed scheme has good service merits.
\end{abstract}

Keywords: sensor; network simulator; congestion; data accuracy; energy efficiency; quality of services (QoS)

\section{INTRODUCTION}

A wireless sensor network (WSN) is deployment of one or more sink nodes and numerous of sensor nodes scattered in a wireless field, which collectively participate to perform a desired task[1]. A wireless sensor network is a physical node having sensing and transmission capabilities which integrates information through sensor computing and wireless communication processing. Sensor node generated data is propagated through adjacent neighboring nodes to the main base station. A multimedia enabled wireless sensor network (WMSN) is a specialized application of (WSN) which have senor capabilities along with multimedia device capabilities, e.g., a minute microphone, battery, audio or video transceiver; The (WMSN) have the equipment and have responsibility to manage the transceiver any multimedia data like still images, video or audio streams, along with responsibility to monitor and sensing data[2]. The resources involved with (WMSN) are power supply, bandwidth, memory, buffer size and processing capability. Since multimedia data having bulky and its transmission requires with high transmission rates and further it requires the ability to process this huge volume of data, but the high traffic of data transfer causes congestion in (WMSN). This adversely degrades the quality of service (QoS) of (WMSN) functionalities. The limitation related to any (WSN) is power, bandwidth, high traffic and large scale scattered deployments covers some basic challenges for managing and designing of (WSN). Such challenging issues demands some energy aware protocol designs that would match with every networking protocol layers. Efficient use of sensor energy is the prime design consideration in developing protocols and these algorithms related to sensor networks predominated (WSN) the research field. Furthermore, (WMSN) in real-time applications which requires both non-delay and throughput for processing the time-critical data to sink within crucial time limit and bandwidth requirements without any loss. Hence, energy awarded sensor networks relating different network layers need efficient resource utilization and effective access technology for sensor readings[3]. In (WMSN), data packets are used to carry and transmit information is done via a hop-by-hop process. The transmission priorities for any heterogeneous (WMSN) differ in between real and non-real time transmission. Real-time transmission has major concerns related to delay and jitter, but is more tolerant to packet losses. Transmission rates needs to be controlled in real-time transmission with highest priority, but non-real-time transmission involves a dynamic queue management method to minimize traffic congestion in network[4]. Various works has been done to maintain (QoS) while (WSN) transmission with loss packet probability, less delay and increased throughput. While a transmitting data through the wireless network, stabilizing its (QoS) is need to satisfy network user expectations. Energy consumption is also an important issue in regard to wireless networks[5]. Various methods has been proposed for using sensor in multimedia infrastructure based on a fuzzy logic to insure (QoS) and energy consideration[6]. Here, we have proposed a fuzzy logical controller (FLC) to manage energy efficiently and minimized the traffic congestion for data transmission of (WMSN)[7].

\section{RELATED WORKS}

Extending network life timing with the minimum energy consumption in wireless sensor networks has been always a challenging task. Most of the energy of exhausted in data transmission within network from data origination nodes to base stations. Recent years researchers giving importance to fuzzy logic control system to manage energy consideration in (WSN). The fuzzy based controller enhances the network power lifetime with effective selection strategy for certain network constraints like node population, traffic density, residual energy, node down and so on. The three most important parameters like network longer lifetime sustainability, minimum energy consumption while 
transmission time and low computational overhead decides the performance of the (WSN).

A selection strategy embedded with the traditional fuzzy logic algorithm is proposed in which minimize transmission path and maximize residual energy concerns up to a satisfied level[8]. The approach focus on enhance residual energy utilization of a node so it would substance for long lasting network lifetime. Inspired through generalized fuzzy logic based approach some dimensions are being opened for energy aware routing algorithms for (WSN)[9]. And this simple and generalized approach is find to be elastic, flexible, robust and reliable. Hence the approach contains sensor networks each node having some different kinds of sensor ability and different power management schema.

The fuzzy set not only persist the network lifespan but also extends the sensor coverage area of the network so it could be extended over larger distance[10]. The approach estimate the adjacent nodes distance between for each sensor nodes needed to transmit data to base one. Based on the priority index, nodes could migrate from each in order to increase the network coverage more area for target field. For the improvement purpose energy of efficiency and load balancing power having unequal clustering node network scheme based on fuzzy logic is presented[11].

Life span is crucial concern which is directly affects straight the energy concerning protocols implied for WSN and those which uses fuzzy logic for heterogeneous node cluster environment are kept in priority. In these type of cluster schema head selection is centralized, but the data collection criteria is shared which lack some (QoS) during transmission[12] [13].

In efficient algorithm design a fuzzy logic control based for energy purpose in hierarchical heterogeneous clustering (WSM) is proposed to solve the clustering and node localization problem[14]. Inspired from the fuzziness a Fuzzy clustering approach also being proposed in order to evaluate determine the optimal number of clusters nodes could participate for (WSN)[15]. Energy reduction analysis for sensor network project that the stability could region holds for long period if finest selections of number of clusters nodes are done in advance[16].

Although, the fuzzy clustering management techniques provides a better optimal solution in determination of shortest path delivery with smart energy utilization schema so that network stabilized for longer duration and central cluster nodes would not struggle to the process of data transmission.

\section{FUZZY LOGIC MODEL}

A fuzzy logic system (FLS) is unique branch of mathematics which has ability deals with numerical and linguistic data. This maps the nonlinear an input data (properties) vector form to the scalar output data, i.e., it is a mapping of numbers into numbers.

Fuzzy set theory and fuzzy logic combines to handle the specifics of the nonlinear data mapping. The (FLS) starts with some crisp set data and dual logic develop to non crisp data. The fuzzy control basis function could be transformed into very powerful reasoning method. Since, their functions could be derived from numerical data or linguistic knowledge and both could further transform in (If-Then-Else rules) form that is easy understandable for human being, as shown figure 1.

Fuzzy logic rule based system for processing heterogeneous distributed wireless sensor network is shown below in Figure 2.

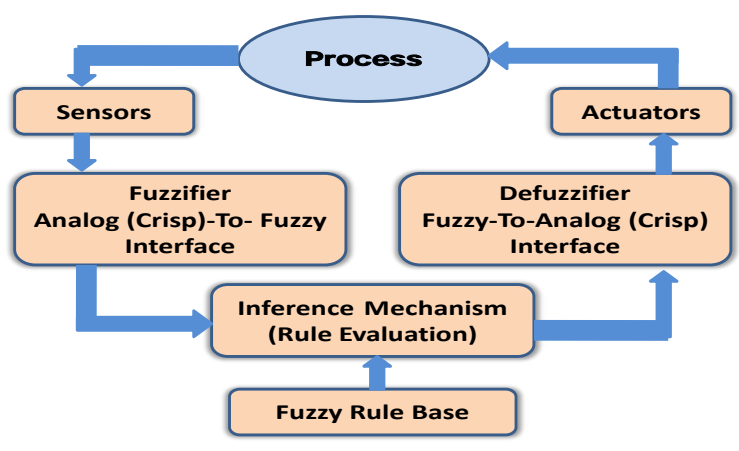

Figure 1. Block Digram of Fuzzy Logic System.

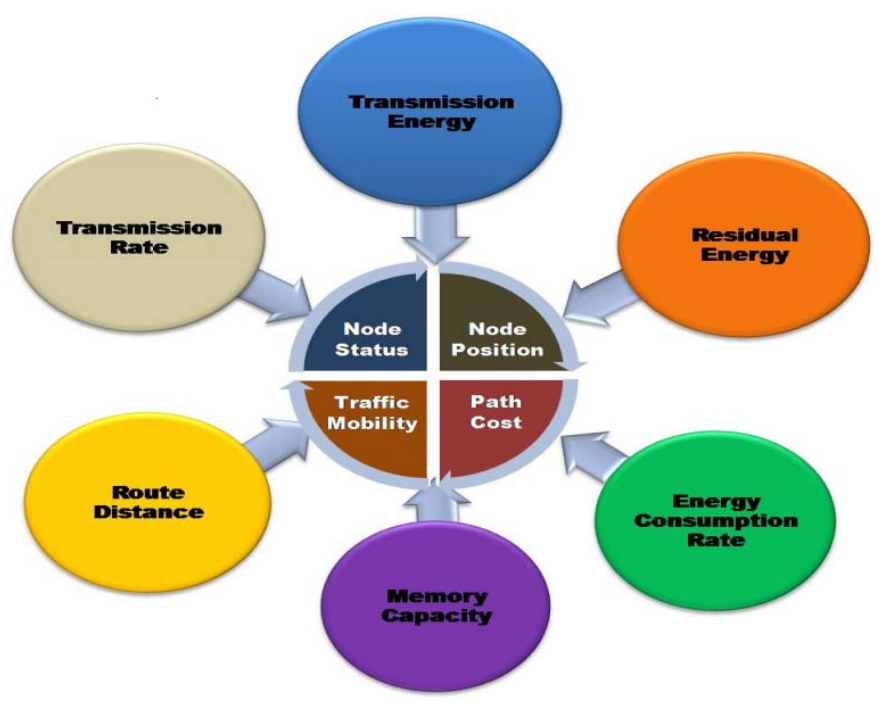

Figure 2. Fuzzy Logic Rule Based System.

Table I. Colour Assingment for Nodes Based on Criteria.

\begin{tabular}{|c|c|c|c|c|c|c|c|}
\hline $\begin{array}{l}\mathbf{S} \\
\mathbf{N} \\
\mathbf{0}\end{array}$ & 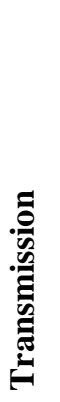 & 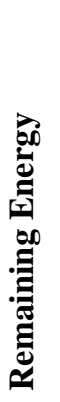 & 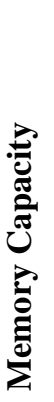 & 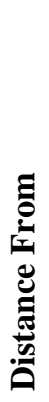 & 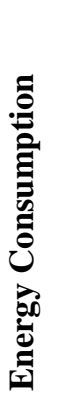 & 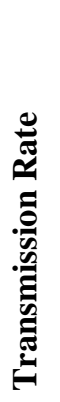 & $\begin{array}{l}\check{0} \\
\dot{0} \\
\dot{y} \\
\dot{z}\end{array}$ \\
\hline 1. & $\mathbf{H}$ & $\mathbf{H}$ & $\mathbf{H}$ & $\mathbf{H}$ & $\mathbf{H}$ & H & Red \\
\hline 2. & $\mathbf{H}$ & H & H & $\mathbf{L}$ & $\mathbf{L}$ & $\mathbf{L}$ & Blue \\
\hline 3. & $\mathbf{L}$ & $\mathbf{L}$ & $\mathbf{L}$ & H & H & H & Green \\
\hline 4. & $\mathbf{H}$ & H & $\mathbf{L}$ & $\mathbf{L}$ & $\mathbf{H}$ & H & Yellow \\
\hline 5. & $\mathbf{L}$ & $\mathbf{L}$ & $\mathbf{H}$ & H & $\mathbf{L}$ & $\mathbf{L}$ & Pink \\
\hline 6. & $\mathbf{H}$ & $\mathbf{L}$ & H & $\mathbf{L}$ & $\mathbf{H}$ & L & Brown \\
\hline 7. & $\mathbf{L}$ & H & $\mathbf{L}$ & H & $\mathbf{L}$ & H & Gray \\
\hline 8. & $\mathbf{L}$ & $\mathbf{L}$ & $\mathbf{L}$ & $\mathbf{L}$ & $\mathbf{L}$ & $\mathbf{L}$ & Black \\
\hline
\end{tabular}

The sensor nodes in network are first categorized according to their functionalities and installation at appropriate numbers within required locations; the surrounding neighboring nodes are organized to form some cluster groups. Inside every cluster 
group among a node that having uppermost energy level could act as cluster node head. On the basis of sensor nodes assigned job and work load capability along with having power level (high: $\mathrm{H}$ or low: L), they have assigned some specific color which is shown in Table 1.

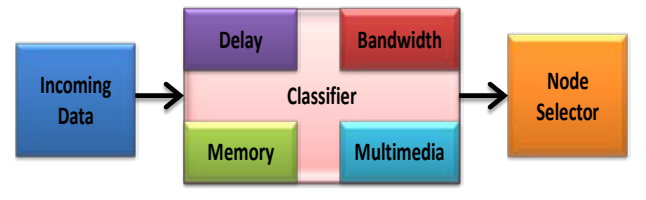

Figure 3. Ant Path Based Route Optimization of Incoming Data Stream.

The given algorithm category classifies the incoming data streams on background of their processing demand requirements for network bandwidth, delay, memory, energy and traffic density. The classification of (MSNW) sensor generated and process data through proposed algorithm is shown in Figure 3.

\section{Energy AWAREd Routing MOdule}

The energy aware routing estimate the power concerning schema that optimize the clusters operation processing depending upon residual energy exhausted in for probabilistic distance estimation along with energy workout in distribution network.

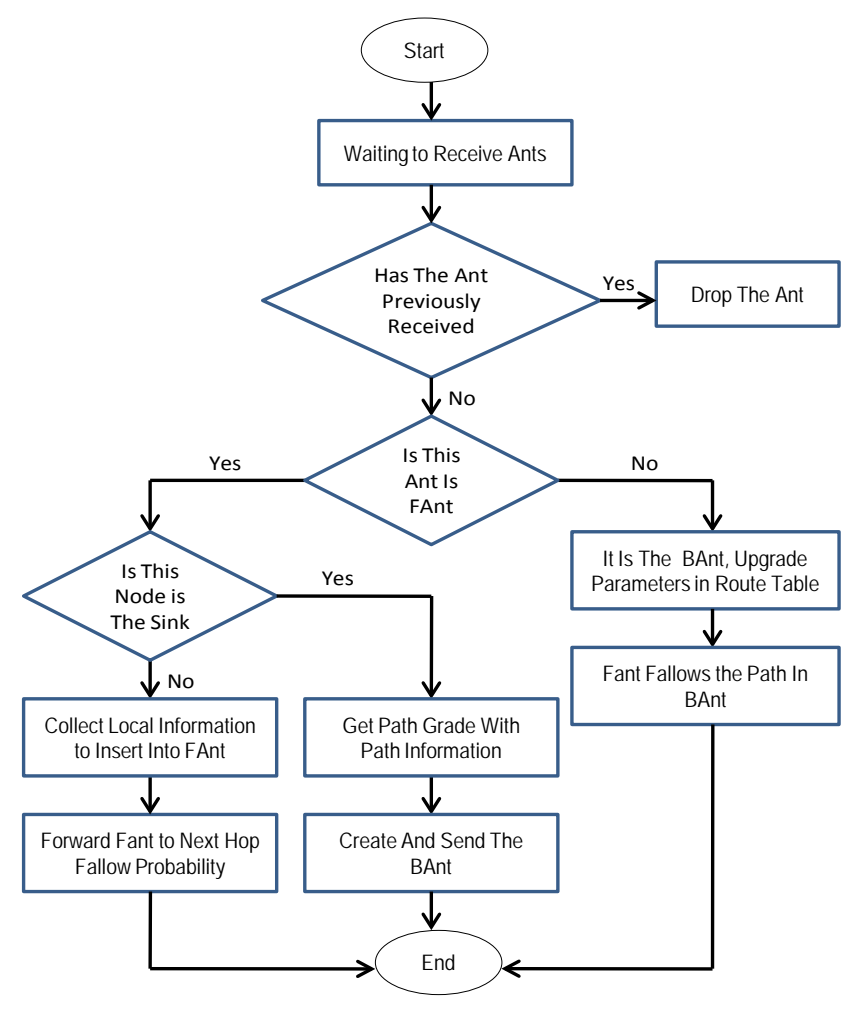

Figure 4. Energy Efficient Route Searching by AntSensNet Algorithm.

Path being analyzed and evaluated through fuzzy logic control maintenance which decides the dynamic path estimation inspired from biologically as an ant search the path with optimization process. Our proposed (QoS) routing algorithm such as AntSensNet for (WMSN) based on an Ant Colony optimization (ACO) algorithm. This dynamic routing algorithm has ability to handle various types of traffics and adjust according to applications requirement[17]. The nodes participate as group in clustering units uses specific surrogate agent process to help in decision channel in heterogeneous environment. In this routing compose of innovative and self learn productive processes. In energy aware path innovative process aimed for traffic classes deployed in (WMSN) and algorithm could choose an optimized path with ensuring (QoS). Multimedia data are transmitted through this optimal path. As sensor network deployed and starts working in each session of life span the path continuously evaluated and updated in a innovative way. This proposed routing algorithms based on (ACO) and digital particle tracks for heterogeneous distributed clustered (WMSN) is show in figure 4 that performs energy aware data transmission among its nodes.

\section{SECURITY Module}

In this section, one way hashing function technique is implemented for ensuring the data security while transmission. This hashing operation is performed for elevation of data to guarantee data integrity and ensures the data packets are unaltered by any intruders during its transmission period. The whole cryptographically operation is defined below.

1) Encryption $\boldsymbol{E}_{P k B S}(\boldsymbol{P I D} \| \boldsymbol{R S N})$.

At beginning the source sensor process ID (PID) and the random seed number (RSN) is concatenated together and then their resultant it is encrypted by public key of base station (PkBS).

2) Encryption $\boldsymbol{E}_{\text {SkSR }}(\mathbf{M D A T A})$.

The sensed multimedia data (MDATA) is encrypted with the symmetric key which one is shared between both sender and receiver (SkSR) to improve confidentiality.

3) Hashing $\boldsymbol{E}_{\boldsymbol{H} k \mathrm{SR}}\left(\boldsymbol{E}_{\mathrm{SkSR}}(\boldsymbol{M D A T A})\right)$.

The resultant encrypted data is treaded by one-way hash function by hash key shared between both sender and reciver which ensures the data integrity.

4) Concating $E_{S k S R}(M D A T A) \| E_{H k S R}\left(E_{S k S R}(M D A T A)\right)$.

The finally obtained encrypted media contains is further concatenated with the previous result obtain in step 2 and step 3.

At the receiver end decryption is performed to re-obtain the original data.

5) Encryption $\boldsymbol{E}_{\boldsymbol{R} k B S}(\boldsymbol{P I D} \| \boldsymbol{R S N})$.

At end the source sensor process ID (PID) and the random seed number (RSN) is concatenated and then their resultant it is decrypted by private key of base station (RkBS).

\section{SIMULATED WORK}

In this section simulation outcomes is analyze and compared with power sustainability within network life span. The accuracy of our proposed fuzzy based routing algorithm for this heterogeneous distributed clustered wireless network is verified with other predictive target tracking ability algorithm. The compression result shows the enhance performance over the parameters like network lifespan, power utilization, traffic congestion, data integrity and security. The (MWSN) is simulated on Network Simulator (NS2) platform which environment is suitable for experimenting network simulation research purpose. For simulation, 1000 nodes are scattered random manner over $(10000 \mathrm{~m} * 10000 \mathrm{~m})$ chosen area, the node have different power capacity and sensing ability. The node organized and form distributed cluster group in which some are source and sink node. Both algorithm (ITPA) and (DPTVA) are compaired and comparison evaluated on basis of 
associated metric total data packets received at base node without transmission delay and traffic congestion, network lifespan and security concerns of the total received data packets. The simulation generated graph are shown in Figure 4, Figure 5 and Figure 6 that proves that proposed schema have better performance result than pervious methods.

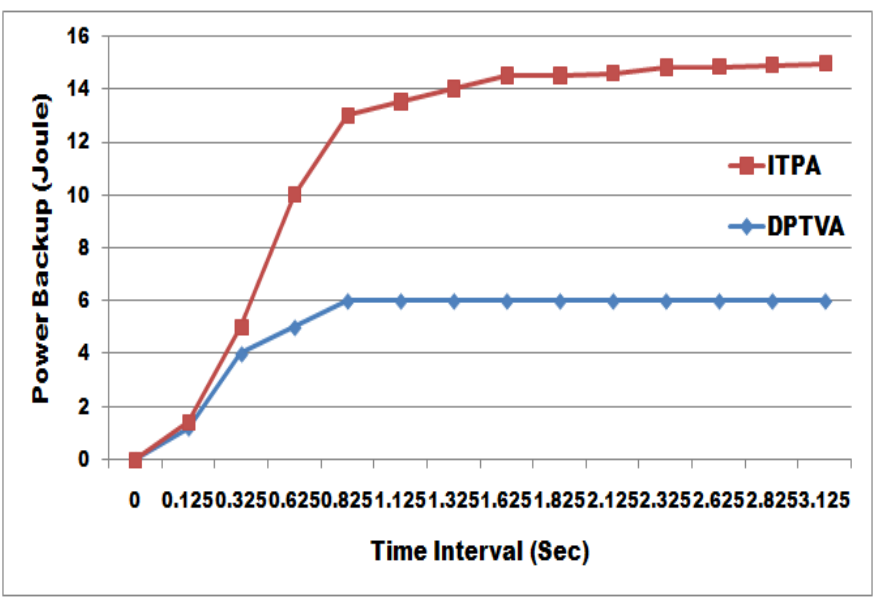

Figure 5. Power Consumption Vs Time.

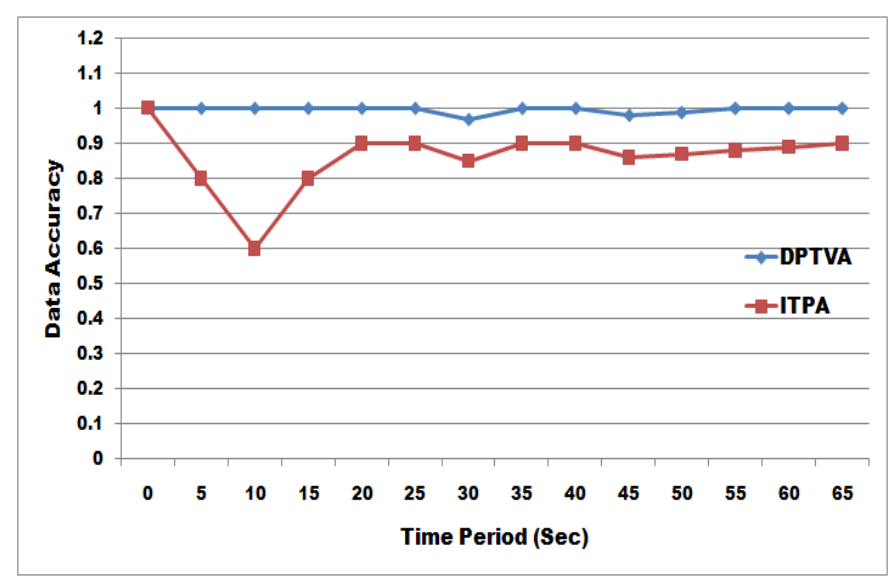

Figure 6. Data Accuracy Vs Time.

\section{CONCULSION}

For heterogeneous distributed (WMSN), network power lifespan and resource utilization critical importance in performance role. Sensing and transmission data combination requires the energy while congestion exhausts most of energy. The paper proposes a noble algorithm for a multimedia sensors network based on fuzzy logic schema for tracking and surveillance remotely mobile target. These techniques optimize the spectral metrics such as network lifespan, energy consumption, transmission rate and data security under heavy stress load on each. Here we applied fuzzy rule based node classification which separates incoming data into different stream and effectively routes according to nodes energy efficiency. This reduces the stress on energy required by working node to overcome the congestion latency of data delivery. The aggregation and hash function compressed various data packets which reduce the transmission overload and ensure their security. Thus, the sensor generated data's are associated and transmitted with full proof security having energy consideration of network.

\section{REFERENCES}

[1] J. Ben-Othman and B. Yahya, "Energy efficient and QoS based routing protocol for wireless sensor network", Journal of Parallel and Distributed Computing 70 (8) (2010) 849-857.

[2] .F. Akyildiz, T. Melodia, and K.R. Chowdhury, "A survey on wireless multimedia sensor networks", Computer Networks 51 (4) (2007) 921-960.

[3] B. Yahya and J. Ben-Othman, "Towards a classification of energy aware MAC protocols for wireless sensor networks", Journal of Wireless Communications and Mobile Computing 9 (12) (2009) 1572-1607.

[4] S. Floyd and V. Jacobson, "Random early detection gateways for congestion avoidance", IEEE/ACM Transactions on Networking 1 (4) (1993) 397-413.

[5] C. Wang, K. Sohraby, M. Daneshmand, and Y. Hu, "Upstream congestion control in wireless sensor networks through crosslayer optimization", IEEE Journal on Selected Areas in Communications 5 (4) (2007) 786-795.

[6] I. Chamodrakas and D. Martakos, "A utility-based fuzzy TOPSIS method for energy efficient network selection in heterogeneous wireless networks", Applied Soft Computing 11 (4) (2011) 3734-3743.

[7] Y.L. Chen, Y.S. Lin, J.W. Wang, and J.H. Wen, "Combined fuzzy-based power control with window-based transmission rate management in multimedia CDMA cellular systems", International Journal of Electronics and Communications 65 (4) (2011) 377-383.

[8] S. Dasgupta and P. Dutta, "An improved Leach approach for Head selection Strategy in a Fuzzy-C Means induced clustering of a Wireless Sensor Network", IEMCON 2011, organised by IEM in collaboration with IEEE on 5-6 $6^{\text {th }}$ of Jan, 2011, pp. 203208, 2010.

[9] T. Haider and M. Yusuf, "A Fuzzy Approach to Energy Optimized Routing for Wireless Sensor Networks" The International Arab Journal of Information Technology, Vol. 6, No. 2, April 2009.

[10] B. J. Farahani, H. Ghaffarian, and F. Mahmood, "A Fuzzy Based Priority Approach in Mobile Sensor Network Coverage”, International Journal of Recent Trends in Engg., Vol. 2.1, Nov 2009.

[11] M. Song and Z. Cheng-lin, "Unequal clustering algorithm for WSN based on fuzzy logic and improved ACO", Elsevier Science Direct on Posts and Telecommunications, Dec 2011.

[12] M. A. Alim, Yucheng Wu, and Wei Wang, "A Fuzzy Based Clustering Protocol for Energy-efficient Wireless Sensor Networks", Proceedings of the 2nd International Conference on Computer Science and Electronics Engineering, 2013.

[13] A. Wilbik and J. M. Keller, "A Fuzzy Measure Similarity Between Sets of Linguistic Summaries”, IEEE Transactions on Fuzzy Systems, VOL. 21, NO. 1, February 2013.

[14] S.K. Swapna, M. K Nanda, and V.S. Sheeba, "Fuzzy Logic based Energy Efficient Hierarchical Clustering in Wireless Sensor Networks", International Journal of Research and Reviews in Wireless Sensor Networks (IJRRWSN) Vol. 1, No. 4, December 2011.

[15] A. S. Raghuvanshi, S. Tiwari, R. Tripathi and N. Kishor, "Optimal Number of Clusters in Wireless Sensor Networks: An FCM Approach”, IEEE International Conference on computer and communication Technology, September 2010.

[16] S. Duraisamy, R. Priya, and V. R. VijayaKumar, "Secured Energy Optimization for Wireless Multimedia Sensor Networks using Fuzzy logic”, International Journal of Computer Science and Mobile Computing, Vol.3 Issue.3, March- 2014, pg. 867-872.

[17] Alexandre Massayuki Okazaki and Antonio Augusto Frohlich, "Ant-based Dynamic Hop Optimization Protocol: a Routing Algorithm for Mobile Wireless Sensor Networks”, IEEE 2011 Jointly organized by workshop SCPA, 2011 and SaCoNAS 2011, Brazil, pp. 1179-1183. [34] Rinju Mariam Rolly and Poornima S., "Base Station Switching Based on Traffic Load Analysis", International Journal of Advance Foundation and Research in Computer, Volume 1, Issue 6, pp. 45-49, June 2014, ISSN 2348 - 4853. 


\section{Author's ProfiLe}

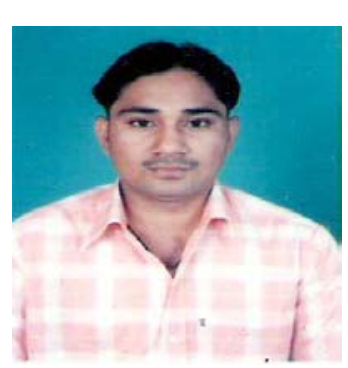

Avinash Singh is M.Sc. Computer Science, M.Tech. and M. Phil. and pursuing Ph.D. in the Department of Computer Science DDU Gorakhpur University, Gorakhpur (U.P. India) under the supervision of Dr. U.N. Tripathi. The area of research interest is Database Security, Networking. Mr. Avinash Singh has published 27 papers in different national and international Conferences/ Journals.

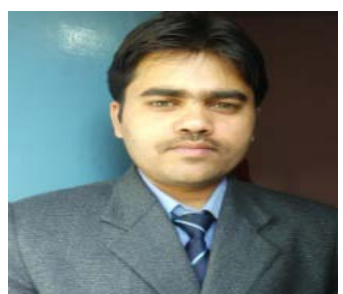

Dr. Surya Pratap Singh is MCA, UGC-NET qualified and Ph.D. from the department of Computer Science DDU Gorakhpur University, Gorakhpur (U.P. India).. The area of research interest is Database Security, Networking. Dr. Surya Pratap Singh has published more than 30 papers in different national and international
conferences/Journals.

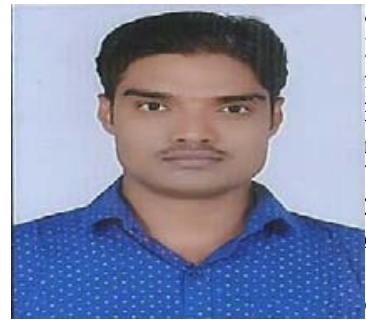

Ir. Arvind Kumar Maurya is MCA and JGC-NET qualified and pursuing Ph.D. In ne department of Computer Science DDU iorakhpur University, Gorakhpur (U.P. ndia) under the supervision of Dr. U.N. ripathi. The area of research interest is )istributed Database Security, Networking. Irvind Kumar Maurya has published 5 apers in different national and international onferences/Journals

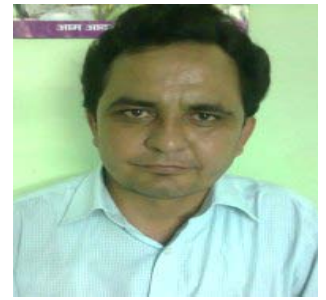

Dr. Upendra Nath Tripathi is Associate Professor in Department of Computer Science DDU Gorakhpur University, Gorakhpur (U.P. India). He has 15 years of teaching and research experience. He has published 52 papers in various National and International Journals/ Conferences. His area of research interest is database systems, networking. 